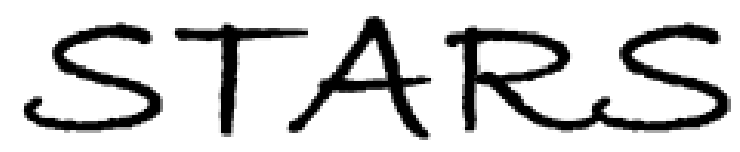

University of Central Florida

STARS

$1-1-1992$

\title{
Electron-Temperature Measurement In Laser-Produced Plasmas By The Ratio Of Isoelectronic Line-Intensities
}

\author{
R. S. Marjoribanks \\ M. C. Richardson \\ University of Central Florida \\ P. A. Jaanimagi \\ R. Epstein
}

Find similar works at: https://stars.library.ucf.edu/facultybib1990

University of Central Florida Libraries http://library.ucf.edu

This Note is brought to you for free and open access by the Faculty Bibliography at STARS. It has been accepted for inclusion in Faculty Bibliography 1990s by an authorized administrator of STARS. For more information, please contactSTARS@ucf.edu.

\section{Recommended Citation}

Marjoribanks, R. S.; Richardson, M. C.; Jaanimagi, P. A.; and Epstein, R., "Electron-Temperature Measurement In Laser-Produced Plasmas By The Ratio Of Isoelectronic Line-Intensities" (1992). Faculty Bibliography 1990s. 526.

https://stars.library.ucf.edu/facultybib1990/526

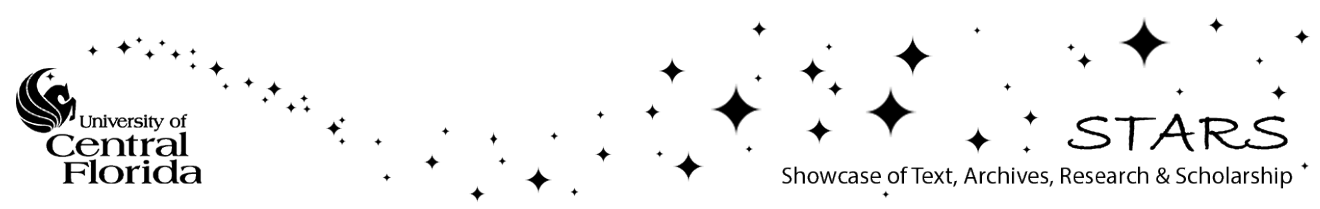




\title{
Electron-temperature measurement in laser-produced plasmas by the ratio of isoelectronic line intensities
}

\author{
R. S. Marjoribanks* \\ Department of Physics, University of Toronto, Toronto, Ontario, Canada M5S IA7 \\ and Ontario Laser and Lightwave Research Centre, 60 St. George Street, Toronto, Ontario, Canada M5S IA7 \\ M. C. Richardson* \\ Center for Research in Electro-Optics and Lasers, University of Central Florida, 12424 Research Parkway, \\ Orlando, Florida 32826 \\ P. A. Jaanimagi and R. Epstein \\ Laboratory for Laser Energetics, University of Rochester, 250 E. River Road, Rochester, New York 14627-1299
}

(Received 27 January 1992)

\begin{abstract}
A class of spectroscopic line ratios has been adapted as a diagnostic of electron temperature from $<100 \mathrm{eV}$ to $>1 \mathrm{keV}$. The diagnostic makes use of the ratio of line intensities from isoelectronic states of different elements in specially prepared targets. The diagnostic is simple to interpret, shows weak dependence on plasma density, requires only low to moderate spectral resolution, uses a single charge state, and can be adapted to minimize line reabsorption and wavelength coincidences with other spectral lines. We present theoretical and experimental results.
\end{abstract}

PACS number(s): $52.70 . \mathrm{La}, 32.30 . \mathrm{Rj}, 52.25 .-\mathrm{b}$

Measurements of temperature in dense regions of laser-produced plasmas [1] typically depend on one of two spectroscopic techniques: the ratio of lines produced in sequential charge states of a single species or the slope of continuum emission.

Measurement of the slope of continuum emission can be difficult in high- $Z$ plasmas or others for which a pure continuum spectrum is difficult to obtain. Line-ratio diagnostics of temperature have different difficulties: Fundamentally, the ratio can be complex to interpret, cross sections or rates may not be well established, or there may be significant contributions from coincidental or blended lines; practically, this may include dynamic range limits in comparing lines of very different intensity, resolution limitations in resolving unrelated close-lying lines, and difficulties in evaluating whole-line intensities where line shapes differ or where there are significant contributions from far-lying line-shape wings.

Using the ratio of lines which are isoelectronic in origin simplifies interpretation and offers concrete advantages in experimental use. In this paper we outline the technique, illustrate the simplifications, give modeling results of the dependence of the ratio on temperature and density, and demonstrate its experimental application in examples of time-integrated and time-resolved spectroscopy. A more detailed comparison with other, established, temperature diagnostics is the subject of a planned followup paper [2].

In astrophysical plasmas, the ratio of lines produced by species of different atomic number is often used to infer the relative abundance of constituent elements, consistent with a model of the distribution of electron temperature and density in the plasma. The approach is reversed here: Laser-plasma targets are constructed to have a thin radiating layer comprised of a known ratio of two elements whose atomic number differs only slightly, chosen according to the anticipated temperature of the plasma, and the line intensities from isoelectronic transitions of the different species provide a diagnostic of the plasma conditions. Typical combinations include $\mathrm{KCl}$ or $\mathrm{NaF}$, which provide a well-mixed layer with a fixed stoichiometry, or $\mathrm{Ti}-\mathrm{V}$ or $\mathrm{Ti}-\mathrm{Cr}$ deposited in a fixed ratio using an alloy sputter block, and analyzed after deposition to verify the proportions.

The elements of the thin layer, having comparable ionization energies, reach similar but not identical distributions of charge state during irradiation of the target. Isoelectronic ionization states of these two elements then have analogous electron configurations, and the ions differ only in their nuclear charge $Z$. For our purposes, the wave functions of two nearly stripped ions may be nearly equivalent mathematically when described in distance and energy parameters rescaled by the nuclear charge $Z$. Consequently, calculations rescaled in energy or radius (e.g., where these enter the cross sections for excitation or ionization) should produce similar results.

Steady-state coronal model calculations of relative abundance of $\mathrm{H}$-like $\mathrm{Na}$ and $\mathrm{Si}$ show abundances expressed as a function of the dimensionless parameter $T_{e} / \chi_{Z}$ (electron temperature rescaled by the ionization energy) produce similar curves [3]; the electron temperature can be expressed as an energy, and the ionization energy written as $Z^{2} \chi_{\mathrm{H}}$, which demonstrates the atomicnumber scaling. Similar curves result for the distribution of excited states of each ion. In Fig. 1(a), this scaled similarity is illustrated for several level populations of hydrogenlike and heliumlike $\mathrm{K}$ and $\mathrm{Cl}$.

Thus the ratio of intensities of lines produced from transition between identical configurations of isoelectronic 

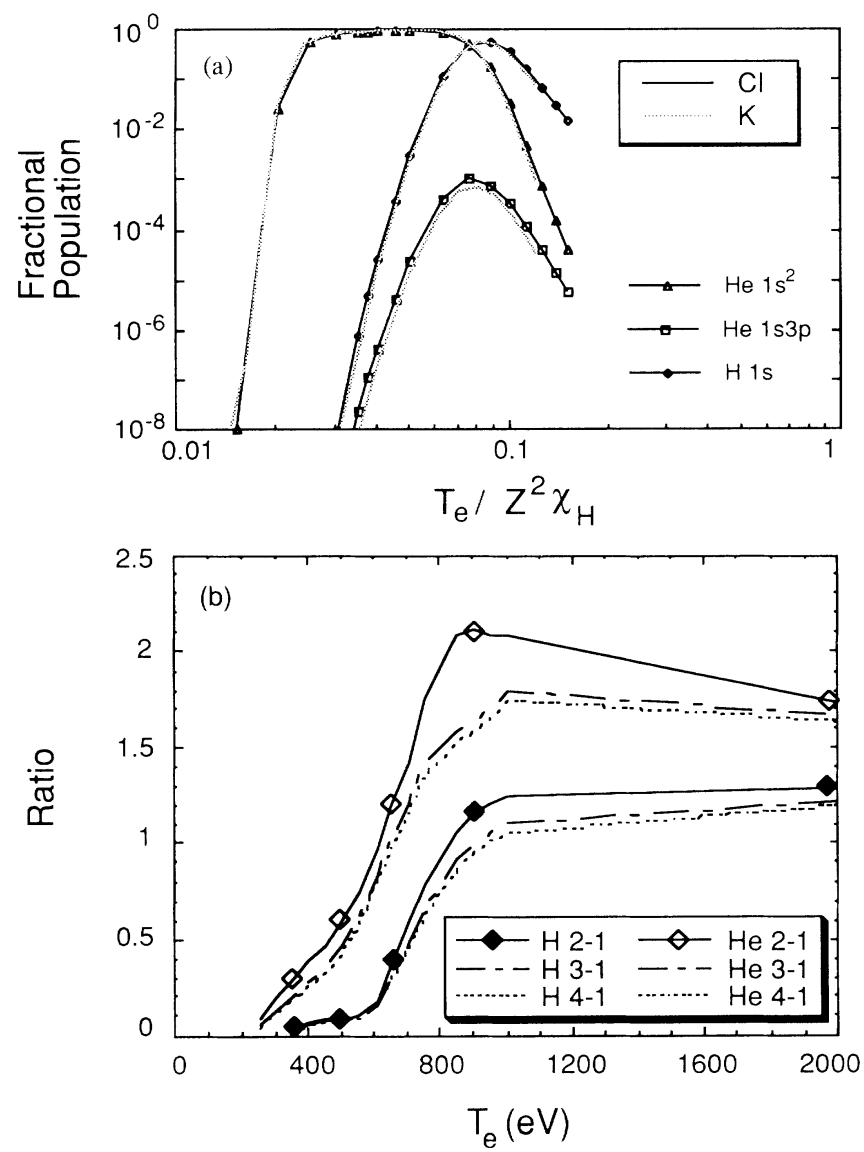

FIG. 1. (a) Rescaling by the ionization energy for the population distributions for $\mathrm{K}$ and $\mathrm{Cl}$. (b) Ratio of isoelectronic $\mathrm{H}$ like and $\mathrm{He}$-like resonance line intensities from $\mathrm{Ti}-\mathrm{V}$ plasma as a function of electron temperature $\left(N_{e}=5 \times 10^{22} \mathrm{~cm}^{-3}\right.$; initial layer thickness $0.08 \mu \mathrm{m}$; opacity included).

states of the two elements produces a useful measure of the temperature of the plasma. Since the lines compared have identical origins, any errors made in identifying or quantifying the population mechanisms are assured to enter both numerator and denominator with the same functional dependence, and generally cancel to lowest order. This tends to make the error in the ratio an order less than the error in contributions, and the technique is somewhat less sensitive to modeling than one using disparate lines.

Calculations of the line ratios of different two-element plasmas were made using RATION, a steady-state rateequation and line-shape solver, with opacity, for detailed treatment of $\mathrm{H}$-like, He-like, and Li-like ion stages, developed at Lawrence Livermore National Laboratory by Lee and others [4]. This code was used to calculate the excited-state distributions of the isoelectronic species, and line ratios were calculated from spontaneous emission rates. We have made calculations for ratios of isoelectronic lines from two-element plasmas composed of $\mathrm{NaF}$, $\mathrm{KCl}$, and a Ti-V alloy. The choice of the average $Z$ of the pair of elements determines the general temperature of suitability; the difference in $Z$ helps establish the temperature range and sensitivity of the diagnostic. Figure 1(b) illustrates RATION modeling for a $\mathrm{Ti}-\mathrm{V}$ plasma with electron density around $5 \times 10^{22} \mathrm{~cm}^{-3}$. This modeling simulates the thin-layer targets used experimentally, described below, and includes opacity effects by preserving the layer areal density.

Figure 2 illustrates the dependence of this technique on plasma electron temperature and density, for the ratio of optically thin lines $1 s^{2}{ }^{1} S_{0}-1 s 3 p^{1} P_{1}$ in vanadium and titanium. In experiments, opacity of the thin signature layer (atomic areal density $\sim 5 \times 10^{17} \mathrm{~cm}^{-3}$ ) makes some difference at higher temperatures and density.

These ideas were tested in several experiments using different two-element targets. Polystyrene spheres of diameter 180-300 $\mu \mathrm{m}$ were prepared, coated with more than $10 \mu \mathrm{m}$ of Parylene $(\mathrm{CH})$, a thin-layer inclusion of different two-element materials $(\mathrm{KCl}, \mathrm{Ti}-\mathrm{V})$ of thickness $0.08-0.15 \mu \mathrm{m}$, and finally a Parylene overcoat of 2-6 $\mu \mathrm{m}$. Targets were irradiated uniformly by the 24-beam OMEGA laser [5] $(\lambda=351 \mathrm{~nm})$ at intensities of $(3-8) \times 10^{14} \mathrm{~W} \mathrm{~cm}^{-2}(\sim 30 \%$ rms variation $)$. Spectra were recorded with time-integrated $\mathrm{Ge}$, mica, and potassium acid phthalate (KAP) Bragg crystal spectrographs onto film, and with a planar mica crystal spectrograph coupled with an $x$-ray streak camera.

Figure 3 is the time-integrated spectrum of a thinlayered $\mathrm{Ti}-\mathrm{V}$ alloy target (15454) prepared by sputtering $\mathrm{Ti}$ and $\mathrm{V}$ from an alloy sputter block prepared in the ratio $\mathrm{Ti}: \mathrm{V}=3: 7$; $\mathrm{x}$-ray fluorescence measurements of the target after preparation show the sputtered target retained the Ti-V ratio of the sputter block. The $80-$ nm-thick Ti-V layer was located $2 \mu \mathrm{m}$ deep in a $\mathrm{CH}$ target, as described above. Irradiated at $7.9 \times 10^{14} \mathrm{~W} \mathrm{~cm}^{-2}$, the resulting spectrum shows the $1 s^{2}{ }^{1} S_{0}-1 s 2{ }^{1} P_{1}$ resonance lines of $\mathrm{He}$-like and $\mathrm{Ti}$ and $\mathrm{V}$, together with the close-lying inter-

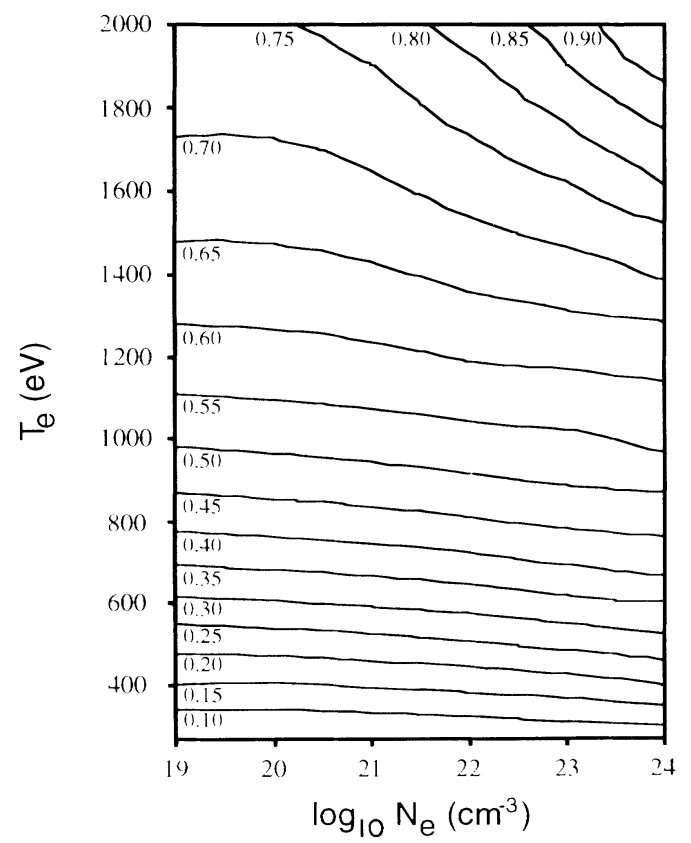

FIG. 2. Contour plot of the intensity of the He-like line $1 s^{2} S_{0}-1 s 3 p^{1} P_{1}$ from $\mathrm{V}$ divided by that of $\mathrm{Ti}$, for optically thin lines (RATION). 


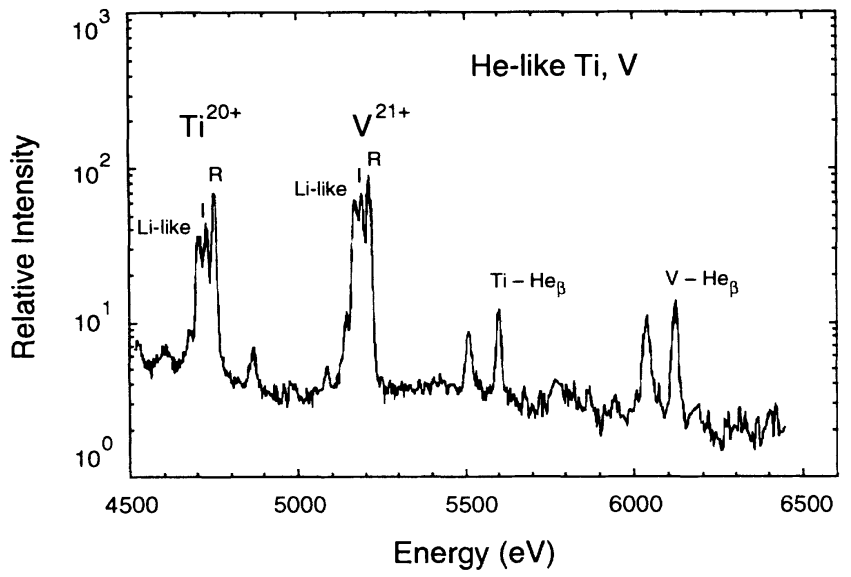

FIG. 3. Time-integrated spectrum from Ti-V alloy thin signature-layer target includes He-like $1 s^{2}{ }^{1} S_{0}-1 s 2 p^{1} P_{1}$ resonance $(R)$, intercombination $(I)$, and Li-like multiplet of $\mathrm{Ti}$ near $4750 \mathrm{eV}$, and the $\mathrm{Ti} 1 s^{2}{ }^{1} S_{0}-1 s 3 p{ }^{1} P_{1}$ line ("He ${ }_{\beta}$ ") near $5580 \mathrm{eV}$; isoelectronic lines in $\mathrm{V}$ are visible at 5200 and 6120 eV. Also visible are long-wavelength satellites to the 1-3 resonance lines. The ratio of $\mathrm{Ti}$ to $\mathrm{V}$ in the target was $3: 7$ in order to balance line intensities.

combination and satellite lines, and the respective $1 s^{2}{ }^{1} S_{0}-1 s 3 p{ }^{1} P_{1}$ resonances. Ratios from the resonance and intercombination lines and of the intercombination and satellite lines indicate a time-integrated and intensity-history-weighted density between mid-1022 $\mathrm{cm}^{-3}$ and about $10^{23} \mathrm{~cm}^{-3}$. The $1 s^{21} S_{0}-1 s 3 p^{1} P_{1}$ resonance lines are certainly optically thin in this case, and their ratio yields a time-integrated electron temperature of about $600 \mathrm{eV}$.

A similar Ti-V shot (15412) was recorded with a planar-crystal streak spectrograph [6]. This instrument affords a spectral resolving power $\lambda / \Delta \lambda \geq 1000$, and a time resolution of about 15 ps. The $1 s^{2}{ }^{1} S_{0}-1 s 3 p^{1} P_{1}$ lines of He-like $\mathrm{Ti}$ and $\mathrm{V}$ were streaked, and Fig. 4(a) shows their ratio. An independent optical fiducial, recorded alongside the x-ray data, established the time $t=0$ of the peak of the irradiating laser pulse [7].

Figure 4(b) gives the temperature history inferred from the measured ratio in Fig. 4(a), using the data of Fig. 1(b). In fabricating the targets, the Ti-V alloy ratio (3:7) was chosen in anticipation of the relative ionization, so as to bring the two He-like line intensities near to equal values, simplifying quantitative reduction of streak camera data. For comparison, these figures include the intensity history of the $\mathrm{Ti}$ line used in the ratio, showing burnthrough before the peak of the laser. At times after the peak, the line ratio and inferred temperature of the ionizing layer increase slightly, as is also measured in companion experiments using the time-resolved ratio of the $1 s^{2} S_{0}-1 s 2 p^{1} P_{1}$ resonance to close-lying Li-like satellite lines [1]; this may be due to continued heating as the layer joins the coronal plasma. It may also indicate changing population mechanisms in the late-time recombing plasmas-explicitly time-dependent modeling is now being conducted with a recent, fully time-dependent, version of RATION to evaluate both the recombining phase and
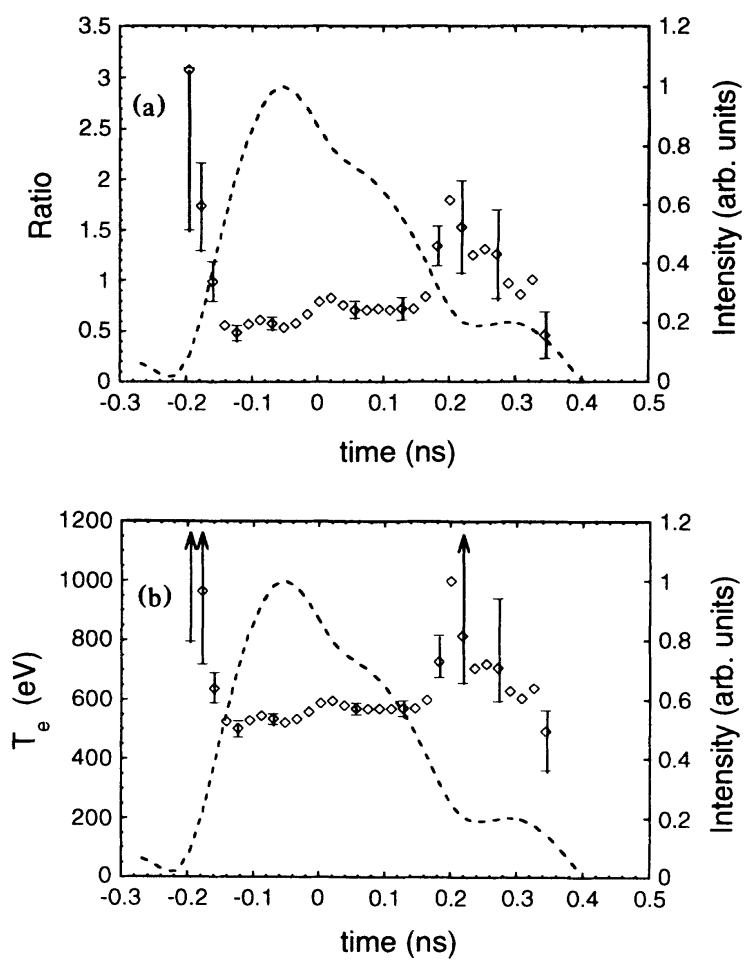

FIG. 4. (a) Time-resolved isoelectronic line ratios for $1 s^{2}{ }^{1} S_{0}-1 s 3 p^{1} P_{1}$ lines of $\mathrm{Ti}$ and $\mathrm{V}$, as above. Data shown have been corrected for the 3:7 alloy ratio. (b) Time-resolved temperatures deduced from (a). The intensity history of the Ti line above is included (dashed line), illustrating the increased error bars for small signals at early and late times. Time 0 marks the peak of the laser pulse, determined by a streaked optical fiducial pulse (not shown).

early-time ionization.

The current diagnostic approach can be of particular use when using high-throughput, low-resolution spectroscopy such as that provided by a transmission grating spectrograph. Where resolution is not sufficient to resolve individual lines useful for temperature measurement, the two-element method may be applied to unresolved multiplets of isoelectronic lines for each element, where the multiplets are themselves resolved from each other. We have recorded such emission from a transmission-grating streak spectrogram of a $\mathrm{SiO}_{2}$ target with known amounts of $\mathrm{Na}$. Unresolved multiplets of $\mathrm{H}$ - and $\mathrm{He}$-like lines and recombination continuua were distinguishable as being from $\mathrm{Si}, \mathrm{Na}$, and other target elements. Analysis of the unresolved isoelectronic features then showed that the temperature of the emission zone of this particular shot rose during irradiation from about 350 to roughly $500 \mathrm{eV}$.

It is useful to recall that rather than measuring true thermodynamic temperature, any line ratio technique provides a parametrization of the electron energy distribution, which may itself not be Maxwellian, i.e., the statistical temperature of the diagnostic populations is not necessarily identical with any thermodynamic temperature. Any ratio characterizes the electron distribution over the range of electron energies which contribute to the populations of the two radiating states. Like any ratio technique, 
the isoelectronic ratio temperature may parametrize a particular region of the distribution; with the flexibility of choosing lines from different elements, it is important to choose elements of similar enough $Z$ that the isoelectronic lines of each ion sample the electron distribution comparably.

Commonly, the ratio of resonance lines from two highlying levels in generalized local thermodynamic equilibrium with the next ionization state is used as temperature diagnostic. Typically, two such lines of comparable intensity lie too close to one another in energy to provide good temperature resolution by their ratio. Additionally, such high-lying lines may be affected by ionization lowering at high electron densities. In general, the two-element isoelectronic technique we describe offers particular advantages as a spectroscopic diagnostic temperature, as follows. (1) Interpretation of the ratio is simplified by considering isoelectronic lines, since the mechanisms controlling the two line intensities and line shapes are the same. (2) Intense lines of relatively simple or well-characterized origin can be used. (3) Since the same population mechanisms will contribute comparably to both lines, calculational errors will typically be somewhat reduced in the ratio, rather than typically being compounded. (4) The technique is broadly applicable and adaptable over different temperature ranges, using different pairs of target materials. (5) Many lines become available in this technique, choosing to suit a particular diagnostic spectral range or resolution, or plasma opacity condition, or to avoid overlap with emission or absorption lines from other target elements. (6) Control of the stoichiometry of elements in the target can be used to control a priori relative line intensities, in order to improve quantitative reduction, or to accommodate limitations in dynamic range by making intensities the same to zeroth order.

The authors would like to thank R. W. Lee for generously making the code RATION available for independent use, G. Kulcsar and F. W. Budnik for code reductions, G. Gregory and F. Marshall for their experimental advice, and S. Noyes for target fabrication. This work was supported in part by the U.S. Department of Energy Office of Inertial Confinement Fusion under Agreement No. DE-FC03-85DP40200 and by the Laser Fusion Feasibility Project at the Laboratory for Laser Energetics, which is sponsored by the New York State Energy Research and Development Authority and the University of Rochester. Additional support for part of this work was provided by the Natural Sciences Research Council of Canada, and by the Ontario Laser and Lightwave Research Centre.
*Work performed in part while the author was at the Laboratory for Laser Energetics, University of Rochester, 250 E. River Rd., Rochester, NY 14627-1299.

[1] See, e.g., R. L. Kauffman et al., Phys. Rev. A 35, 4286 (1987); B. K. F. Young et al., Phys. Rev. Lett. 25, 2851 (1988); O. Willi et al., Phys. Rev. A 39, 6090 (1989).

[2] R. S. Marjoribanks, F. W. Budnik, M. C. Richardson, P. A. Jaanimagi, R. Epstein, and N. Delamater (unpublished).

[3] G. Bekefi, C. Deutsch, and B. Yaakobi, in Principles of Laser Plasmas, edited by G. Bekefi (Wiley, New York, 1976), p. 549.

[4] An early version is described in R. W. Lee, B. L. Whitten, and R. E. Strout, II, J. Quant. Spectros. Radiat. Transfer
32, 91 (1984).

[5] J. Bunkenburg et al., IEEE J. Quantum Electron. QE-17, 1689 (1981).

[6] M. C. Richardson, G. G. Gregory, R. L. Keck, S. A. Letzring, R. S. Marjoribanks, F. J. Marshall, G. Pien, J. S. Wark, B. Yaakobi, P. D. Goldstone, A. Hauer, G. S. Stradling, F. Ameduri, B. L. Henke, and P. A. Jaanimagi, in Laser Interaction and Related Plasma Phenomena, edited by H. Hora and G.H. Miley (Plenum, New York, 1986), Vol. 7, p. 179.

[7] P. A. Jaanimagi, L. DaSilva, G. G. Gregory, C. Hestdalen, C. D. Kiika, R. Kotmel, and M. C. Richardson, Rev. Sci. Instrum. 57, 2189 (1986). 